\title{
Indentation : fondamentaux et développements
}

\author{
Alex Montagne ${ }^{1}$, Didier Chicot ${ }^{2}$, Alain Iost $^{1}$ et Eric Le Bourhis ${ }^{3, a}$
}

Du 12 au 14 octobre 2016, à l'École Nationale Supérieure d'Arts et Métiers (Lille), s'est tenu le colloque Indentation 2016, sixième édition de ce congrès francophone organisé tous les deux ans. Le colloque a réuni tous les scientifiques utilisateurs avancés des techniques d'indentation instrumentée. Ces techniques d'indentation statique ou dynamique sont utilisées largement par l'industrie pour caractériser les surfaces de matériaux fonctionnalisés. Ces techniques récentes ne sont pas encore au stade des essais conventionnels de caractérisation des matériaux massifs. C'était justement l'objet du colloque Indentation 2016 : faire un état des lieux des aspects fondamentaux (compréhension de la mécanique complexe des essais localisés) et des développements (notamment in-situ) en cours dans le domaine.

Durant ce colloque, qui a réuni 120 participants issus des équipes françaises et francophones possédant des équipements instrumentaux et/ou des moyens numériques dédiés aux techniques d'indentation, environ une trentaine de présentations orales et une vingtaine de posters ont été présentés. L'ensemble de ces travaux présentés, lors de 7 sessions a suscité de nombreuses discussions et échanges entre les participants. Pour la première fois, une formation sur l'indentation a été organisée la veille du colloque (http://conference.mines-douai.fr/indentation2016/ programme-formation/) avec plus de 80 participants dont de nombreux doctorants et industriels. Fort de ce succès, cette formation sera reconduite lors de la prochaine édition à Liège en Belgique.

Chaque édition du colloque a donné lieu à un numéro spécial dans la revue Matériaux \& Technique [1-5]. Inscrit dans cette tradition, nous présentons dans ce numéro thématique, un ensemble d'articles issus d'une sélection de contributions au colloque, publiés après un processus d'évaluation et de révision. Ces articles offrent un aperçu de toutes les thématiques abordées au cours du colloque. L'ensemble du numéro regroupe des travaux fondamentaux et appliqués et permet de faire un point sur des sujets d'actualité dans le domaine de l'indentation.

Les éditeurs (A. Montagne, D. Chicot, A. Iost et E. Le Bourhis) tiennent à remercier les membres du comité d'organisation (T Coorevits, I Hervas, A Iost, A Montagne, A Van Gorp, A Thuault, A Tricoteaux, D Betrancourt, G Louis, P Pizette, D Chicot, X Decoopman, F Roudet) pour la tenue de ce colloque, ainsi que les membres du comité scientifique du groupe indentation multiéchelle (E. Barthel, S. Benayoun, D. Chicot, J.-P. Guin, V. Keryvin, E. Le Bourhis, J.-L. Loubet, G. Mauvoisin, H. Pelletier, http://www.sf2m.asso.fr/CommissionsThematiques/ Indentation.htm) qui ont accepté de rapporter chacun sur plusieurs articles publiés dans cet ouvrage.

\section{Références}

[1] S. Benayoun, E. Le Bourhis, Matériaux \& Techniques 93 (2005) 185

[2] O. Bartier, G. Mauvoisin, Matériaux 83 Techniques 96 (2008) 9

[3] E. Le Bourhis, G. Mauvoisin, Matériaux \& Techniques 99 (2011) 161

[4] S. Bec, S. Benayoun, Matériaux \& Techniques 101 (2013) 301

[5] H. Pelletier, E. Le Bourhis, Matériaux \&3 Techniques 103 (2015) 601

\footnotetext{
a Auteur de correspondance : Eric.le.bourhis@univ-poitiers.fr

1 MSMP - Mechanics, Surfaces and Materials Processing, École Nationale Supérieure d'Arts et Métiers, 8 Boulevard Louis XIV, 59046 Lille, France

2 Université de Lille - Sciences et Technologies, IUT A de Lille - Dpt GMP, Rue de la Recherche "Le Recueil", BP 90179, 59653 Villeneuve d'Ascq Cedex, France

3 Institut P', UPR 3346, CNRS - Université de Poitiers, Département de Physique et Mécanique des Matériaux, SP2MI Boulevard Marie et Pierre Curie, BP 30179, 86962 Futuroscope Chasseneuil Cedex, France
} 